\title{
Correction to: Inverse spectral theory for semiclassical Jaynes-Cummings systems
}

\author{
Yohann Le Floch ${ }^{1}$ (D) Álvaro Pelayo ${ }^{2}$ San Vũ Ngọc $^{3}$ \\ Published online: 17 July 2019 \\ (c) Springer-Verlag GmbH Germany, part of Springer Nature 2019
}

\section{Correction to: Math Ann (2016) 364:1393-1413 https://doi.org/10.1007/s00208-015-1259-z}

We explain why Theorem B in the original article does not follow from the main result of this paper (Theorem A). While we conjecture that Theorem B should nevertheless be true, in this erratum we prove a slightly weaker version of it.

Theorem B in the original article is not a mere corollary of Theorem A, contrary to what was stated in the original article. Indeed, the proof implicitly uses the assumption that, for a system with a unique focus-focus singularity, the twisting index must be trivial, which does not always hold. In this erratum we point out why the proof of Theorem B is incomplete, and show that a slightly weaker version of Theorem B still holds, which we state as Theorem B' at the end of the erratum. We conjecture that the original Theorem B still holds as is but our proof method is not powerful enough to verify this.

Communicated by Thomas Schick.

The original article can be found online at https://doi.org/10.1007/s00208-015-1259-z.

\footnotetext{
$凶$ Yohann Le Floch ylefloch@unistra.fr http://irma.math.unistra.fr/ lefloch/

Álvaro Pelayo

alpelayo@math.ucsd.edu

San Vũ Ngọc

san.vu-ngoc@univ-rennes1.fr

https://perso.univ-rennes1.fr/san.vu-ngoc/

1 Institut de Recherche Mathématique Avancée, Université de Strasbourg, 7 rue René Descartes, 67000 Strasbourg, France

2 Department of Mathematics, University of California, San Diego, 9500 Gilman Drive \# 0112 , La Jolla, CA 92093-0112, USA

3 Institut Universitaire de France, Institut de Recherches Mathématiques de Rennes, Université de Rennes 1, Campus de Beaulieu, 35042 Rennes Cedex, France
} 
In order to give more details, we will need to restate some definitions. Once a weighted polygon (a representative of the polygonal invariant) of the system has been fixed, Proposition 5.4 in [1] associates an integer with each focus-focus singularity. We will call this integer the twisting integer, in order to avoid confusing it with the twisting index invariant. As explained in Definition 5.9 of [1], the twisting index invariant is the equivalence class, under a certain group action, of weighted polygons indexed by twisting integers at focus-focus singularities. The point is that, even when there is only one focus-focus singularity, choosing another representative in this equivalence class amounts to changing both the weighted polygon and the associated twisting integer. In other words, these two invariants are not independent; there indeed exists a representative of the polygonal invariant for which the corresponding twisting integer is zero, but one cannot start from any weighted polygon and assume that the associated twisting integer vanishes. A good way to define the twisting index jointly with the polygon invariant is explained in [2].

Consequently, the argument leading to Theorem B still needs to be completed, and we can only conjecture that the original statement is actually correct. Fortunately, it can be amended as follows. The natural idea is to restrict the statement to a smaller class of Jaynes-Cummings systems for which the twisting integer is fixed in advance. However, this is not as simple as it seems, since the twisting integer depends on the choice of weighted polygon representing the polygonal invariant. Hence, the first requirement would be to fix a normalization of this weighted polygon; one can for instance do as follows. It is known [3, Corollary 5.5] that in a semitoric system $(J, H)$ with at least one focus-focus singularity, the function $J$ must have a global minimum or a global maximum $J_{ \pm}$at a point $m \in M$; if it has both, we choose $J_{ \pm}=J_{-}$to be the minimum of $J$. Let $H_{-}$be the minimum of the restriction of $H$ to $J^{-1}\left(J_{ \pm}\right)$. Then $\left(J_{ \pm}, H_{-}\right)$is an elliptic-elliptic critical value of the momentum map $(J, H)$. We first choose an arbitrary representative $\Delta$ of the polygonal invariant and we call the minimal vertex $v$ the vertex of $\Delta$ corresponding to the critical value $\left(J_{ \pm}, H_{-}\right)$. We now claim that there exists a polygon for this system that lies above the horizontal line through its minimal vertex $v$, and in the closest position to this line. Indeed, it follows from [3] that the edges of $\Delta$ starting at $v$ are directed along integral vectors $(a, c)$ and $(b, d)$ such that $\left(\begin{array}{ll}a & b \\ c & d\end{array}\right) \in \operatorname{SL}(2, \mathbb{Z})$. Let $T:=\left(\begin{array}{ll}1 & 0 \\ 1 & 1\end{array}\right)$. Assume that $J_{ \pm}$is a minimum value (the maximum case is treated similarly). Then we must have $a \geqslant 0$ and $b \geqslant 0$. If $J^{-1}\left(J_{ \pm}\right)$is a single point, then no edge of the polygon $\Delta$ can be vertical: $a \neq 0$ and $b \neq 0$ (cases (b) and (c) in Fig. 1). If $J^{-1}\left(J_{ \pm}\right)$is a submanifold of positive dimension, then $(b, d)$ must be vertical (case (a) in Fig. 1), i.e. $b=0$; hence $a \neq 0$ (and necessarily $a=1$ ). Thus, in all cases one has $a>0$. Let $-r$ be the integer part of $\frac{c}{a}$, so that $r$ is the smallest integer such that $r a+c \geqslant 0$. We now select the new representative of the polygonal invariant by applying to $\Delta$ the matrix $T^{r}$ (viewed as an affine map with origin taken at the vertex $v$ ). As a result, the new edges from $v$ are $(a, r a+c)$ and $(b, r b+d)$, see Fig. 1, in which the matrix $\left(\begin{array}{ll}a & b \\ c & d\end{array}\right)$ is: 
(a)
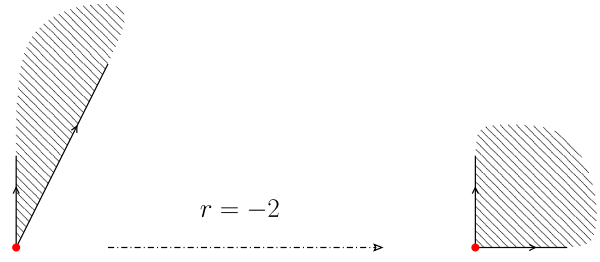

(b)
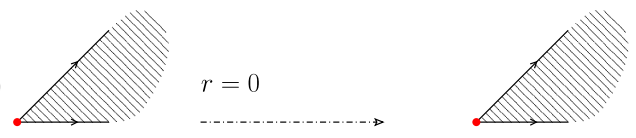

$$
r=1
$$

(c)
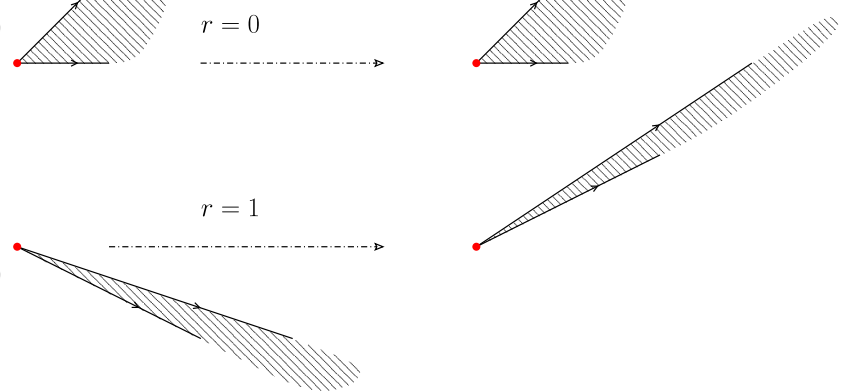

Fig. 1 Normalization of various polygons when $J$ has a global minimum. The red point corresponds to the image of the value $\left(J_{ \pm}, H_{-}\right)$. The integer $r$ indicates that a global transformation $T^{r}$ was applied to the polygon in order to normalize it
(a) $\left(\begin{array}{ll}1 & 0 \\ 2 & 1\end{array}\right)$,
(b) $\left(\begin{array}{ll}1 & 1 \\ 0 & 1\end{array}\right)$,
(c) $\left(\begin{array}{cc}2 & 3 \\ -1 & -1\end{array}\right)$.

If $J^{-1}\left(J_{ \pm}\right)$is a submanifold of positive dimension, the normalized bottom edge is always horizontal $(r a+c=0)$, but in general it can have a positive slope $r+\frac{c}{a} \in[0,1)$.

Definition 0.1 For any semitoric system with at least one focus-focus singularity, we call normalized twisting indices the twisting integers of the focus-focus points, when computed with a normalized semitoric polygon described above.

Note that normalized polygons are not unique in the equivalence class of the polygonal invariant, since one may act on them by the group $G=\{-1,1\}^{m_{f}}$ ( $m_{f}$ being the number of focus-focus points), as described in [1, Formula (4.5)]. The $2^{m_{f}}$ possible semitoric polygons are obtained by "cutting" the image $F(M)$ along vertical half-lines starting at the focus-focus values, going either upwards or downward, and the action of $G$ corresponds to the changes in the polygon when one passes from one choice to the other.

In spite of this, the aforementioned normalized twisting indices are well defined, because the $G$ action preserves the twisting integers, see for instance [1, Formula (5.10)].

In the following statement we only consider the case $m_{f}=1$, since this was our initial motivation in the original article. 
Theorem B' Let $k_{0} \in \mathbb{Z}$ be given. Let $(P, Q)$ be a quantum integrable system of JaynesCummings type on $M$ with normalized twisting index $k_{0}$, and for which the Bohr-Sommerfeld rules hold. Then from the knowledge of $\operatorname{JointSpec}(P, Q)+\mathcal{O}\left(\hbar^{2}\right)$, one can recover the principal symbol $\sigma(P, Q)$ up to isomorphisms of semitoric integrable systems.

Proof From the proof in the original article we first recover the image of the momentum map $F(M)$, which indicates whether $J$ admit a global minimum or maximum (or both). Then we construct a polygon that is a representative of the polygonal invariant and select the minimal vertex $v$. From this, one can obtain the direction $(a, c)$ of the bottom edge at $v$, where $a$ and $c$ are coprime integers, and compute $r=-E(c / a)$. By applying the transformation $T^{r}$, we obtain one of the normalized polygons. Since a normalized polygon is detected and the normalized twisting index is known a priori, the full twisting index invariant is determined. Then the rest of the proof recovers the other four invariants, which finally fully determines the system up to isomorphism.

Note that Theorem B' should also work in the case $m_{f}>1$, but one needs to check the proof in detail and this is not the purpose of this erratum.

Acknowledgements We would like to thank Daniele Sepe for noticing and pointing out the mistake.

\section{References}

1. Pelayo, A., Vũ Ngọc, S.: Semitoric integrable systems on symplectic 4-manifolds. Invent. Math. 177(3), 571-597 (2009)

2. Sepe, D., Vũ Ngọc, S.: Integrable systems, symmetries, and quantization. Lett. Math. Phys. 108(3), 499-571 (2017)

3. Vũ Ngọc, S.: Moment polytopes for symplectic manifolds with monodromy. Adv. Math. 208(2), 909934 (2007)

Publisher's Note Springer Nature remains neutral with regard to jurisdictional claims in published maps and institutional affiliations. 